\title{
Orthodontic management of supernumerary teeth- a case report
}

\section{Rahman MA ${ }^{1}$ BDS, FCPS, Alam MM ${ }^{2}$ BDS, FCPS and Hossain MZ ${ }^{3}$ BDS, PhD}

\begin{abstract}
Supernumerary tooth is one that is additional to the normal series and can be found in almost any region of the dental arch .Mesiodens is the most common type of supernumerary tooth found in the premaxilla between two central incisors. They may be single, multiple, unilateral or bilateral, erupted or unerupted in one or, both jaw. It usually results in oral problems such as malocclusion, food impaction, poor aesthetics, and cyst formation. Early diagnosis and treatment is suggested to prevent orthodontic and pathologic complications. This article highlights the orthodontic management of a case of twenty five year old Bangladeshi male with two supernumeraries- one is a supplemental tooth erupted in the oral cavity causing malocclusion and another impacted mesiodens near the nasal floor. The case was managed by a joint effort of maxillofacial surgeon and orthodontist. At first, maxillofacial surgeon removed the two supernumerary teeth. After healing the orthodontic correction was done with fixed appliance using multiloop edgewise technique. It took nearly 18 months to complete the treatment. The patient was happy with the new appearance and function.
\end{abstract}

Key words: Supernumerary teeth, Mesiodens, supplemental teeth. (Ban J Orthod and Dentofac Orthop, Oct 2011; Vol-2, No. 1, p 30-33)

\section{INTRODUCTION}

Supernumerary tooth is one that is additional to the normal series and can be found in almost any region of the dental arch ${ }^{1}$. Etiology of the development of supernumerary teeth is not clear. Atavism theory suggested that supernumerary teeth were the result of phylogenetic reversion to extinct primates with three pairs of incisors. It may be due to dichotomy of the tooth $\mathrm{bud}^{2}$ or due to localized, independent and conditioned hyper activity of dental lamina ${ }^{3}$. Genetic factor also suggested since many incidence of recurrence within the same family ${ }^{3,4}$

The supernumerary teeth may be single, multiple, unilateral or bilateral, erupted or unerupted and in one or, both jaws. Multiple supernumeraries are rare in individuals with no other associated disease or syndromes. Multiple supernumerary teeth are usually associated with conditions such as cleft of the lip and palate or syndromes like Cleidocranial Dysplasia and Gardner's syndrome. ${ }^{5}$

The prevalence ranges between $0.3-0.8 \%$ in the primary dentition and $0.1-3.8 \%$ in the permanent dentition. Males are affected approximately twice as often as females. ${ }^{1,5}$

Supernumerary teeth are classified according to morphology and location. In the primary dentition, morphology is usually normal or conical. There is a greater variety of forms presenting in the permanent dentition. There are four morphological different types of supernumerary teeth; Conical( most common with triangular or conical- shaped crown and complete root formation, ${ }^{6}$ Tuberculate (Barrel shaped appearance and a crown consisting of multiple tubercles with incomplete or absent root formation ), Supplemental (morphological resemblance of a normal tooth, most often a permanent maxillary lateral incisor) and Odontome (numerous small tooth like structures or a single irregular mass). They are also classified on the basis of location: Mesiodens- present typically in between the maxillary central incisors ,Paramolar- present beside a molar, Distomolar- present distal to the last molar and Parapremolar- in the premolar region. Cases involving one or two supernumerary teeth most commonly involve the anterior maxilla, followed by mandibular premolar region. When multiple supernumeraries are present the most common site is the mandibular premolar. $3,6,7$

The various problems associated with the supernumerary teeth are: failure of the eruption of permanent teeth, or their displacement. They can also cause crowding, loss of tooth vitality due to root resorption and may also be associated with pathology like cyst. Occasionally, supernumerary teeth are not associated with any adverse effects and may be detected as a chance finding during radiographic examination. Early extraction of supernumerary tooth is advocated to reduce treatment time and complications. 8,9

\section{CASE REPORT}

A twenty five year old Bangladeshi male reported to the Department of Orthodontics and Dentofacial Orthopedics, Dhaka Dental College and Hospital, Dhaka with a complaint of irregular upper front teeth. His reason of attendance was to improve his esthetics.
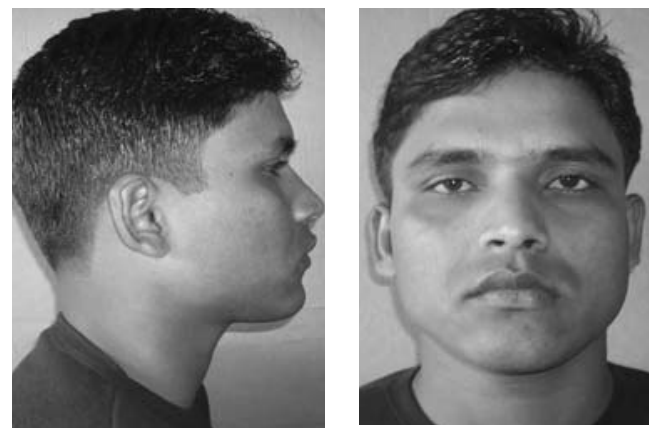

Figure 1: Pre-treatment extra-oral facial photographs 
On extra-oral examination of the patient had a square face form and a straight profile and a pleasant outlook. Intra oral examination revealed a supplemental tooth, which was erupted behind the right upper central incisor and posed obliquely to the incisal edge of the left upper central incisor tooth. Right upper central incisor was proclined over the right lateral incisor. While remaining other teeth were in the normal alignment. He had full set of permanent dentition with class I molar and canine relationship and an overbite and over jet of $1 \mathrm{~mm}$. Midline coincided. Oral hygiene of the patient was good with little staining of teeth.
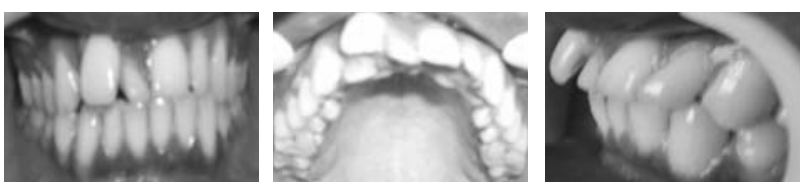

Figure-2: Pre treatment intraoral photograph

A panoramic $\mathrm{x}$-ray confirmed the presence of an impacted mesiodens between the root apices of upper two central incisor tooth and a supplemental tooth along with normal complement of teeth present (Fig. 3a). X-ray lateral cephalogram (Fig. 3b) along with tracing was done as routine orthodontic pre treatment procedure (Table-1).

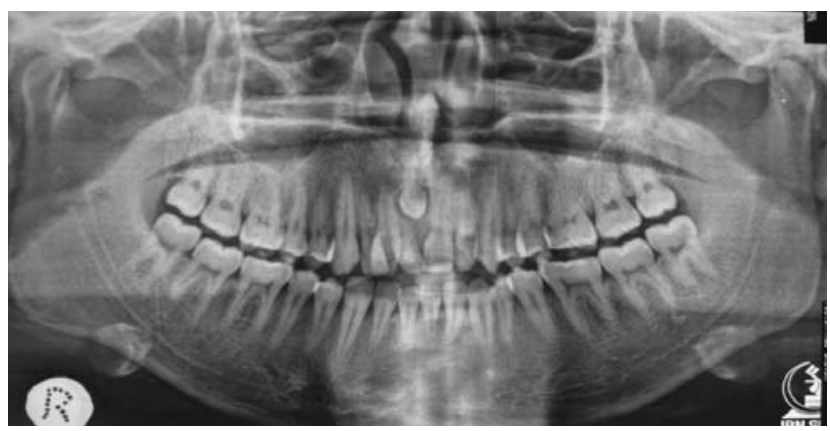

Figure 3a : Pretreatment OPG

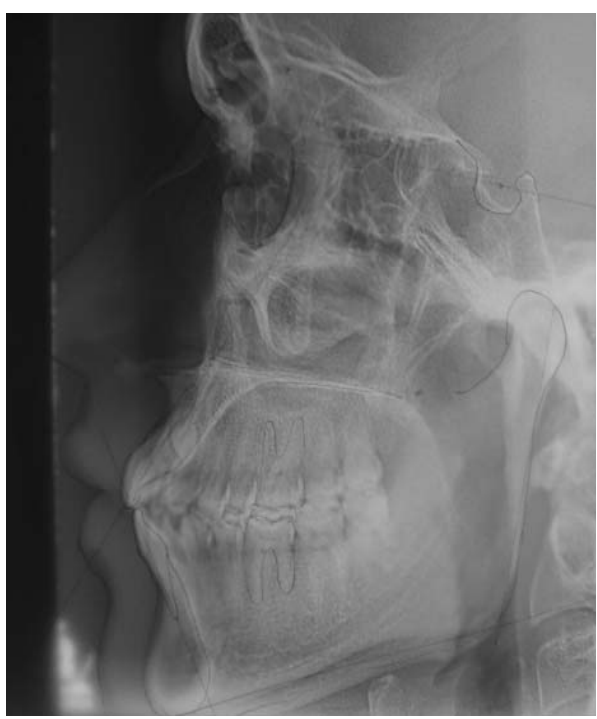

Figure $3 \mathrm{~b}$ : Pretreatment cephalogram

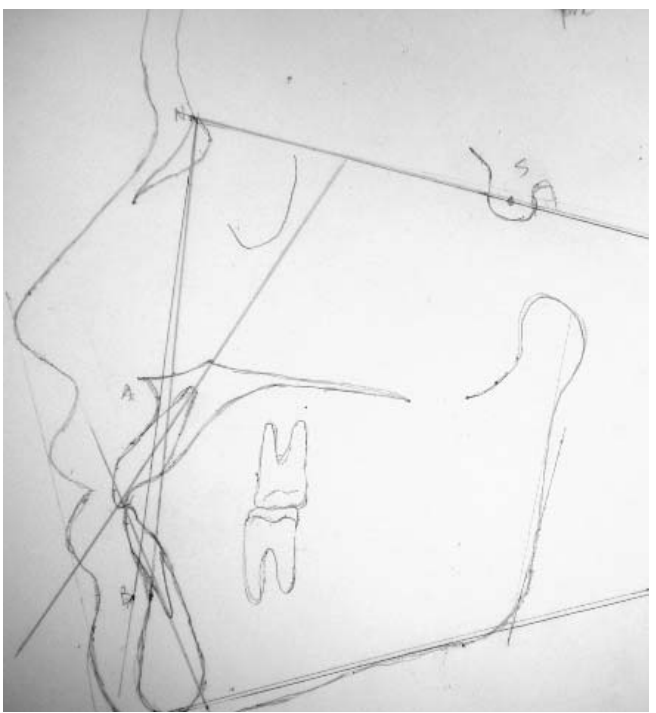

Figure $3 \mathrm{c}:$ Pretreatment ,lateral cephalogram tracing

Table-1: pre treatment lateral cephalometric tracing results

Parameter

$\begin{array}{ll}\text { Reference } & \text { Patient's } \\ \text { Measurement } & 8 \\ \left( \pm 2^{\circ}\right) & \text { Measurement }\end{array}$

\begin{tabular}{lll}
\hline SNA (angle) & $82^{\circ}$ & $83^{\circ}$ \\
SNB (angle) & $80^{\circ}$ & $80^{\circ}$ \\
ANB (angle) & $2^{\circ}$ & $3^{\circ}$ \\
Upper 1 to NA (mm) & $4 \mathrm{~mm}$ & $4 \mathrm{~mm}$ \\
Upper 1 to NA (angle) & $22^{\circ}$ & $23^{\circ}$ \\
Lower 1 to NB (mm) & $4 \mathrm{~mm}$ & $6 \mathrm{~mm}$ \\
Lower 1 to NB (angle) & $25^{\circ}$ & $27^{\circ}$ \\
Inter incisal angle & $131^{\circ}$ & $125^{\circ}$
\end{tabular}

Interpretation-A Class I skeletal jaw relationship with average inclinations of the maxillary and mandibular incisors.
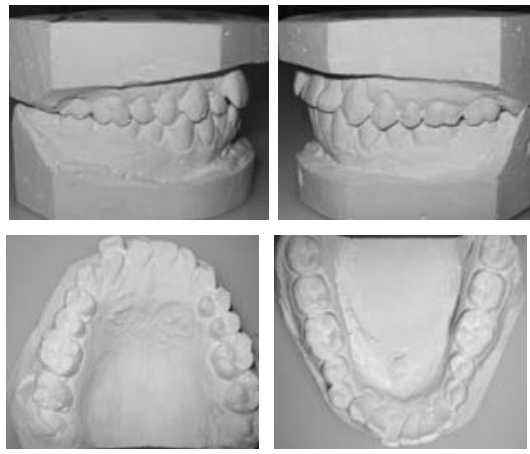

Figure - 4: Pre treatment intra oral photographs of models 


\section{Tooth jaw discrepancy}

Upper arch

Arch perimeter -Total tooth material

$=74 \mathrm{~mm}-79 \mathrm{~mm}$

$=-5 \mathrm{~mm}$

Lower arch

Arch perimeter -Total tooth material

$=63 \mathrm{~mm}-65 \mathrm{~mm}$

$=-2 \mathrm{~mm}$

Pre treatment model analysis shows crowding in both the arches due to tooth jaw discrepancy. He was diagnosed as a case of class I malocclusion with supplemental tooth in between upper central incisor teeth and an impacted mesiodens near the root apices of upper central incisors. A multidisciplinary approach was adopted for the management of the case. The patient was referred to maxillofacial surgeon for extraction of the impacted mesiodens along with the supplemental tooth. After that he was supervised for orthodontic intervention (fig.5)
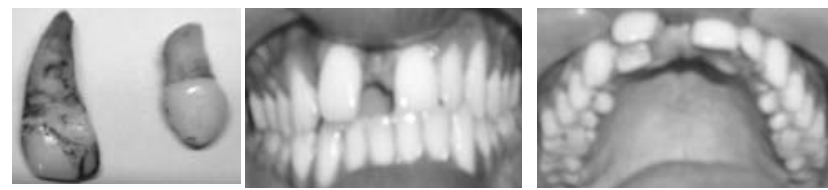

Figure-5: Extracted supernumeraries and post extraction upper jaw photograph

Standard edgewise appliance was fitted and a standard arch wire sequence of 0.014-inch multi loop SS wire. A segmented arch wire of 0.016-inch SS wire with closing loop was incorporated between the upper central incisors. A stop loop was given distal to left upper central incisor. The left side of the arch wire was engaged in bracket channel upto first molar, while on the right side it was engaged only to the central incisor tooth to facilitate its movement without putting pressure on anchor teeth. Once the arches were fully aligned and space was closed, finishing and detailing of teeth was done with 0.016 inch round wires. Following debonding, upper Hawley retainers were used to maintain. The patient was advised to wear the retainers full time for six month, then night-time use might be sufficient as long as he could. He was instructed for a quarterly follow up schedule.
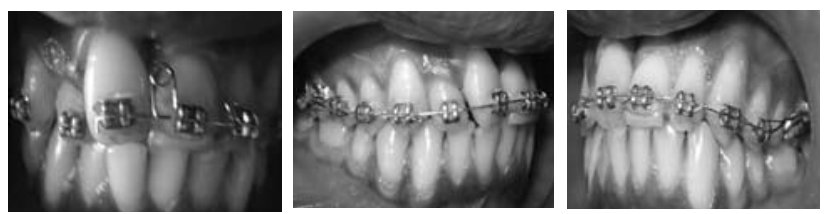

Figure-6: Treatment progression (closing space and detailing occlusion)
Post treatment OPG ( fig.7) shows space closure, root parallelism and absence of supernumeraries.

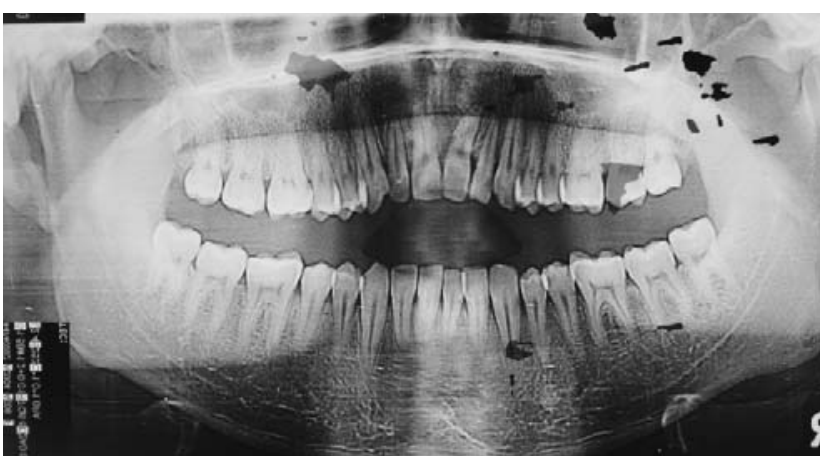

Figure-7: Post treatment OPG

Table-2: post treatment lateral cephalometric tracing results

\begin{tabular}{lll}
\hline Parameter & $\begin{array}{l}\text { Reference } \\
\text { Measurements } \\
\left( \pm 2^{\circ}\right)\end{array}$ & $\begin{array}{l}\text { Patient's } \\
\text { Measurement }\end{array}$ \\
\hline SNA (angle) & $82^{\circ}$ & $83^{\circ}$ \\
SNB (angle) & $80^{\circ}$ & $80^{\circ}$ \\
ANB (angle) & $2^{\circ}$ & $3^{\circ}$ \\
Upper 1 to NA (mm) & $4 \mathrm{~mm}$ & $4 \mathrm{~mm}$ \\
Upper 1 to NA (angle) & $22^{\circ}$ & $23^{\circ}$ \\
Lower 1 to NB (mm) & $4 \mathrm{~mm}$ & $6 \mathrm{~mm}$ \\
Lower 1 to NB (angle) & $25^{\circ}$ & $27^{\circ}$ \\
Inter incisal angle & $131^{\circ}$ & $125^{\circ}$ \\
\hline
\end{tabular}

Post treatment lateral cephalometric tracing shows Class I skeletal pattern with average incisor inclination.
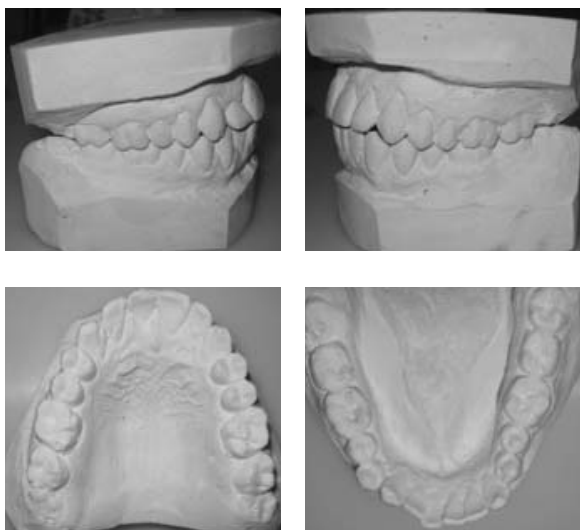

Figure-8: Post treatment intra oral photographs of models 

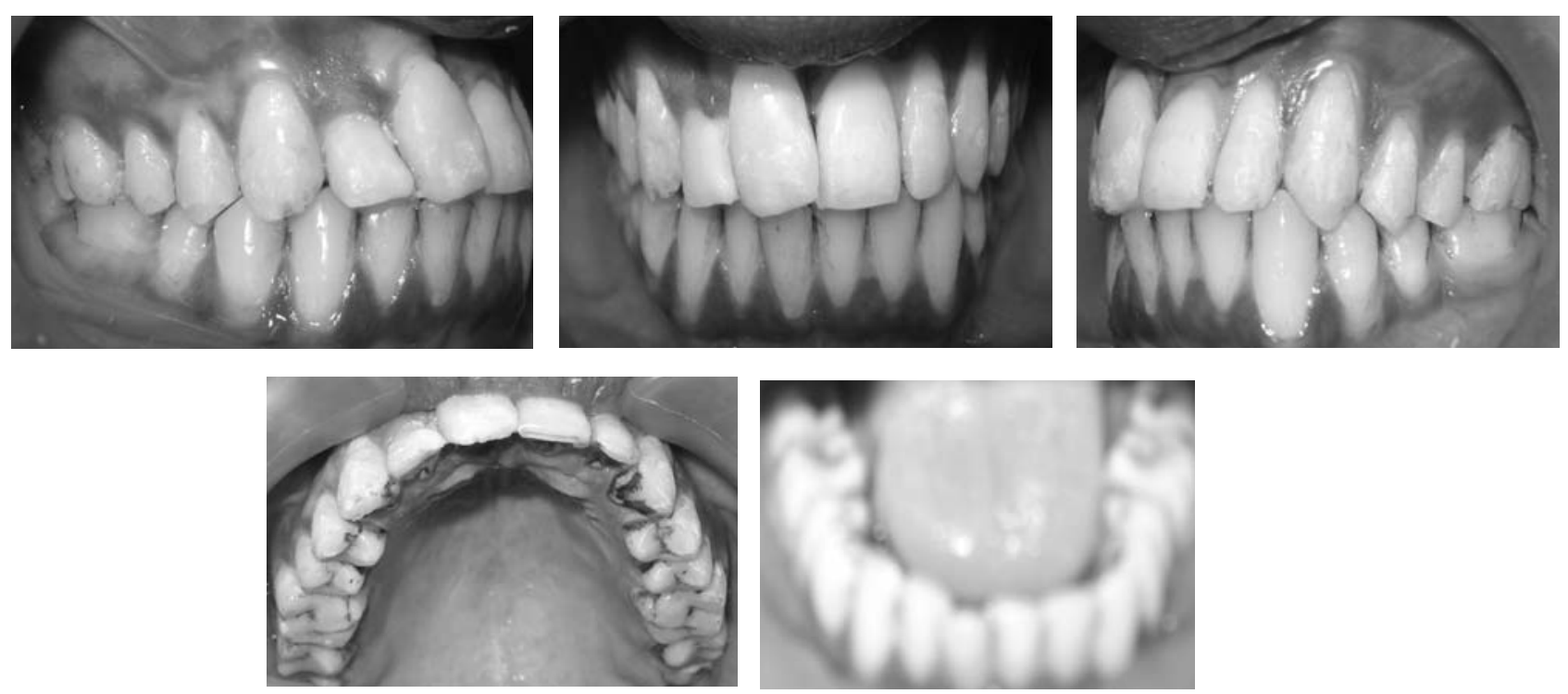

Figure-9: Post treatment intra oral photographs of both jaws

\section{DISCUSSION}

Clinical and radiographic identification of all the teeth is very important for a good treatment planning. The position of the supernumeraries can be ascertained with tube shift method. A precise positioning with surrounding hard and soft tissue relationship is also possible with the aid of a three dimensional Cone Beam Computed Tomography (CBCT) image. ${ }^{3,6,9}$ It may be difficult to formulate an ideal treatment plan for all cases with supernumerary teeth. But an effort can definitely be made. Treatment may vary from extraction of supernumerary teeth or extraction coupled with orthodontic correction to establish a good aesthetic as well as occlusion. ${ }^{2,3}$ The presence of an undiscovered supernumerary tooth, may obstruct orthodontic space closure. In the present case, it was decided to extract the supplemental tooth and the impacted mesiodens for the proper alignment of the teeth. ${ }^{8}$ A multidisciplinary approach was adopted for the management of the case. Regarding space closure a segmented archwire with closing loop mechanics was used. It has the superiority over sliding mechanics in terms of anchorage demand and precise tooth movement. ${ }^{9}$

\section{CONCLUSION}

Early diagnosis and appropriate management can minimize the potential complications caused by supernumerary tooth. Whatever the etiology is, it is the duty of the practitioner to recognize the signs suggesting the presence of supernumerary tooth. Extraction in mixed dentition helps spontaneous alignment of the adjacent tooth. Orthodontic intervention in time is immensely beneficial to patients from esthetic and functional point of view.

\section{REFERENCES}

1. Alberti B, Mondani PM, Parodi V. Eruption of supernumerary permanent teeth in a sample of urban primary shool population in Genoa, Italy. Eur J Peadiatr Dent 2006 jun;7(2):89-92.

2. Gill D S, Tredwin C, Naini F B. Diagnosis and Management of Supernumerary teeth. Dent Update 2008;35:510-20.

3. Meighani G, Pakdaman A. Diagnosis and management of supernumerary(mesiodens) : A review of the literature. Journal of Dentistry. Tehran University of Medical Sciences. Tehran. Iran. 2010;7(1):4149.

4. Leena V, Krishnan G, Sidhi P, Archana A, Navjot S. Mesiodens with an Unusual Morphology- A Case Report. J Oral Health Comm Dent 2009;3(2):42-44.

5. Scheiner M A, Sampson W J. Supernumerary teeth: A review of the literature and four case reports. Australian Dental Journal 1997;42(3):160-5

6. So L.LY. Unusual supernumerary teeth. Angle Orthod 1990;60:28992

7. Kolokitha G O-E, Papadopoulou A K. Impaction and apical root angulation of the maxillary central incisor due to supernumerary teeth: Combined surgical and orthodontic treatment. Am J Orthod Dentofacial Orthop 2008;134:153-60.

8. Sharma A. Familial occurrence of Mesiodens- A case report. J Indian Soc Paedo Pre Dent. 2003;21(2):84-5.

9. Proffit W R. Contemporary Orthodontics. 2008, chapter 12: page 449.

\section{Corresponds to:}

Dr. Md. Anisur Rahman

BDS, FCPS (Orthodontics)

Department of Orthodontics.

Dhaka Dental College, Dhaka 